\title{
Aportación de la formación cívica y ética a la educación en la perspectiva de género
}

\author{
Ibarra Rosales Guadalupe \\ Instituto de Investigaciones sobre la Universidad y la Educación (IISUE) de la Universidad \\ Nacional Autónoma de México, Ciudad de México, México \\ irge@unam.mx
}

\section{Resumen}

En este trabajo se analiza y desarrolla la educación en la perspectiva de género que proporciona el Plan de Estudios titulado Aprendizajes Clave para la Educación Integral (SEP, 2017) del nuevo Modelo Educativo para la Educación Básica (SEP, 2017) elaborado e implementado por la Secretaría de Educación Pública (SEP) de México en ese año.

En este Plan de Estudios se sustenta la educación de primaria y secundaria y forma parte del mismo la asignatura Formación Cívica y Ética que constituye el espacio curricular en el que se incluye la educación en la perspectiva de género con el estudio de la temática Igualdad y Perspectiva de Género. Esta asignatura se imparte tanto en el nivel de la primaria en los grados; $4^{\circ}, 5^{\circ}$ y $6^{\circ}$ como en el nivel de secundaria; $1^{\circ} 2^{\circ} 3^{\circ}$ grados.

La metodología para analizar esta propuesta educativa tuvo como referente teórico los Objetivos de aprendizaje para alcanzar el ODS 5 establecido en la Agenda 2030 elaborados por la UNESCO (2017). En el desarrollo de este trabajo se establecen la complementariedad y las semejanzas encontradas entre los aprendizajes esperados del estudio de la temática Igualdad y perspectiva de género y los Objetivos de aprendizaje para alcanzar el ODS 5 referido a la Igualdad de género.

\section{Abstract}

This paper analyzes and develops education in the gender perspective provided by the Curriculum for Key Learning for Comprehensive Education (SEP, 2017) of the new Educational Model for Basic Education (SEP, 2017) developed and implemented by the Secretariat of Public Education (SEP) of Mexico in that year.

In this Plan of Studies, primary and secondary educations are supported and the Civic and Ethics Training course is part of it, which constitutes the curricular space in which education in the gender perspective is included with the study of the theme Equality and Gender Perspective. This subject is taught at the elementary level in the grades; 4th, 5th and 6th as in the secondary level 1st 2nd 3rd grades.

The methodology used to analyze this education al proposal had as a theoretical reference the Learning Objectives to achieve SDG 5 established in the 2030 Agenda prepared by UNESCO (2017). In the development of this work, complementarily and similarities are established between the lessons learned from the study of Gender Equality and Perspective and Learning Objectives to achieve SDG 5 related to Gender Equality.

Palabras clave: educación, género, igualdad de género, equidad de género.

Keywords: education, gender, gender equality, gender equity.

\section{INTRODUCCIÓN}

En este trabajo se presentan los resultados del análisis de la temática Igualdad y Perspectiva de Género que constituye la propuesta de educación en la perspectiva de género que forma parte del Plan de Estudios para la Educación Básica (SEP, 2017) el cual fue elaborada e implementada por la Secretaría de Educación Pública (SEP) de México en 2017.

La metodología de análisis fue la siguiente a) identificar y desarrollar los componentes curriculares y educativos que articulan y estructuran la temática Igualdad y perspectiva de género (SEP,2017), b) analizar esta temática teniendo como referente los Objetivos de aprendizaje para alcanzar el ODS 5 elaborados por la UNESCO (2017), c) identificar la complementariedad, las semejanzas encontradas entre los aprendizajes esperados del estudio de la temática Igualdad y perspectiva de género con los Objetivos de aprendizaje para alcanzar el ODS 5 que se refiere a la lgualdad de Género. 
Se parte de considerar que la educación en la perspectiva de género en los últimos grados de primaria y en todos los grados de secundaria es importante porque la mayoría de los alumnos se encuentran iniciando o viviendo la adolescencia, la cual es una edad clave para que adquieran un conjunto de conocimientos, valores y actitudes en relación al género que les permitan a mediano plazo ejercer la igualdad y la equidad de género en las relaciones e interacciones que establezcan tanto con mujeres como con hombres.

Mediante la enseñanza de la temática de la Igualdad y Perspectiva de Género (SEP, 2017) los alumnos adquieren conocimientos sustantivos para comprender la problemática del género en la sociedad contemporánea como son los siguientes: el significado sociocultural de género, la importancia y las implicaciones que tiene el ejercicio de la igualdad y la equidad género.

El estudio del significado cultural del género resulta relevante en la edad de la adolescencia porque a través de su análisis es posible que los alumnos comprendan que la categoría de género no se limita a aquellos aspectos y problemáticas ligadas a las mujeres sino queesta categoría se refiere tanto a hombres como a mujeres y constituye el significado sociocultural mediante el cual una sociedad identifica y establece diferencias entre las mujeres y los hombres.

Aquí cabe recuperar la siguiente visión de género: «Por género se entiende las construcciones socioculturales que diferencian y configuran los roles, las percepciones y los estatus de las mujeres y de los hombres en una sociedad» (UNESCO, 2014, p.104).

Esta concepción del género muestra que mediante estesignificado, la cultura de una sociedad establece las características, atributos, papeles, actitudes y prácticas sociales que deben de asumir tanto la mujer como el hombre porque son reconocidas y aceptadas como «propias» y adecuadas para el comportamiento y desarrollo de una mujer y de un hombre en la sociedad.

El género como construcción sociocultural afecta tanto a hombres como a mujeres pero se reconoce que a lo largo de la historia las mujeres han padecido y cargado como una pesada losa este significado cultural que la sociedad les otorgó (UNESCO, 2014). Esto porque con base en los rasgos y características que las definen socialmente han experimentado, las discriminación, la violencia de género, el acoso, la intolerancia y un conjunto de práctica sociales negativas que no pueden ser permitidas ni toleradas en una sociedad que busque el logro de la justicia social.

El estudio del género como construcción sociocultural permite comprender a los alumnos que este significado ha sido construido en una cultura y en un momento histórico y por ello es posible que mediante la educación se pueda desplazar este significado dominante en la sociedad actual y comenzar a construir un nuevo significado sociocultural que rompa con los estereotipos en los que se han encapsulado tanto a las mujeres como a los hombres, pero que ha impactado en mayor medida en el desarrollo y en el trato desigual para con las mujeres.

Por ello se revela como importante que en el estudio de la temática de la Igualdad y Perspectiva de Género también se aborde la igualdad de género porque esta categoría también contribuye a fracturar el significado dominante del género al contemplar la igualdad de derechos y oportunidades tanto para las mujeres como para los hombres. Esto lo establece la UNESCO (2014) en los siguientes términos:

«Para la UNESCO, la igualdad de género significa igualdad de derechos, responsabilidades y oportunidades para las mujeres y hombres y para niñas y niños. Supone que se tenga en cuenta los intereses, necesidades y prioridades de mujeres y hombre, reconociendo la diversidad de los distintos grupos de personas. La igualdad de género es un principio relativo a los derechos humanos, un prerrequisito para un desarrollo sostenible centrado en las personas y un objetivo en sí misma» (UNESCO, 2014, p.12).

En México la igualdad de género está respaldada jurídicamente y se ha traducido en leyes como la Ley General para la Igualdad entre Mujeres y Hombres (INMUJERES, 2017). ONU Mujeres México (2015, p.4) señala que la Convención sobre la Eliminación de Todas las Formas de Discriminación contra la Mujer (CEDAW) considera a esta forma de igualdad de género como igualdad jurídica o igualdad formal. Si bien la educación en la perspectiva de género de esta propuesta curricular, no contempla el estudio de esta legislación específica, si incluye la enseñanza de «programas y leyes nacionales e internacionales que prohíben toda práctica discriminatoria dirigida a impedir o anular el ejercicio de los derechos y la igualdad real de oportunidades para que todas las personas gocen o ejerzan sus derechos» (SEP, 2017, p.181). 
ONU Mujeres (2015, p.4) también precisa que la CEDAW establece que se requieren tomar medidas para lograr que esta igualdad jurídica se traduzca en una igualdad sustantiva o igualdad en los hechos.

«La igualdad sustantiva supone la modificación de las circunstancias que impiden a las personas ejercer plenamente sus derechos y tener acceso a oportunidades de desarrollo mediante medidas estructurales, legales o de política pública» (ONU Mujeres, 2015, p. 4).

En el programa de esta propuesta curricular de educación en la perspectiva de género se precisa que el ejercicio del derecho a la igualdad puede encontrase con obstáculos (SEP, 2017, p 202). Por ello en la enseñanza de la temática Igualdad y perspectiva de género en $3^{\circ}$ grado de secundaria establece en las orientaciones didácticas lo siguiente:

«Se puede analizar, de forma particular, el derecho a la igualdad sustantiva entre mujeres y hombres; revisar las leyes que lo garantizan y la forma como ellos pueden demandar que se cumplan dichas leyes en diversos espacios como la escuela, la familia y la localidad» (SEP, 2017, p. 202).

La equidad de género constituye un planteamiento que también contribuye a desplazar el significado sociocultural dominante de género porque se refiere al trato diferente que han tenido las mujeres a lo largo de la historia para atender sus intereses, cubrir sus necesidades y ofrecerles oportunidades para su desarrollo (UNESCO; 2014, p.106).

\section{LA EDUCACIÓN EN LA PERSPECTIVA DE GÉNERO EN LA FORMACIÓN CÍVICA Y ÉTICA}

La asignatura Formación Cívica y Ética no se encuentra aislada en el Plan de Estudios para la Educación Básica de la SEP (2017), sino que está inserta en el campo de Formación Académica denominado Exploración y Comprensión del Mundo Natural y Social (SEP, 2017, p.327). Asimismo esta asignatura está estructurada por cinco ejes: a) Conocimiento y cuidado de sí, b) Ejercicio responsable de la libertad, c) Sentido de pertenencia y valoración de la diversidad, d) Convivencia pacífica y solución de conflictos, d) Sentido de justicia y apego a la legalidad (SEP, 2017, p. 442).

El eje Sentido de Pertenencia y Valoración de la Diversidad comprende a su vez las siguientes temáticas: a) Valoración de la diversidad, no discriminación e interculturalidad, b) Identidad colectiva, sentido de pertenencia y cohesión social, c) Igualdad y Perspectiva de Género (SEP, 2017, p. 442).

En el nivel educativo de primaria, la temática Igualdad y Perspectiva de Género se enfoca a proporcionar a los estudiantes los conocimientos básicos para conocer y comprender la problemática del género en la sociedad contemporánea como son: el género como construcción sociocultural, la igualdad de género y la equidad de género (SEP, 2017, p. 450)

De acuerdo con la UNESCO (2017), la igualdad de género se encuentra estrechamente vinculada con aspectos como la clase la etnia, la cultura, las tradiciones, etc., propios de distintos grupo social, los cuales han contribuido a fortalecer las diferencias entre los hombres y las mujeres. Estos factores socioculturales han incidido en el trato diferente que viven y experimentan muchas mujeres en la sociedad contemporánea, ya que se traduce en la mayoría de los casos en discriminación. Una discriminación doble puesto que no solo son relegadas y excluidas por el hecho de ser mujeres sino también por el grupo social al que pertenecen.

Desde esta perspectiva la temática titulada Valoración de la Diversidad, no Discriminación e Interculturalidad, hace aportaciones importantes para que los alumnos de primaria adquieran una visión y un conocimiento integral de la problemática del género al enfocarse en los siguientes aspectos: a) permite recuperar y valorar la diversidad sociocultural propia del país, b) aborda y analiza la exclusión o discriminación que pueden experimentar tanto hombres como las mujeres en función del «género, origen étnico, cultural, religioso, condición económica, física y otras», (SEP,2017, p.450). Contempla que los alumnos identifiquen y distingan aquellas «creencias, tradiciones culturales y prácticas que generan intolerancia o distintas formas de exclusión» (SEP, 2017, p. 450).

En el nivel educativo de secundaria la temática de Igualdad y Perspectiva de Género está orientada a lograr que los alumnos asuman la igualdad de género, mediante el ejercicio de la equidad de género 
en las relaciones e interacciones que establezcan, así como la participación en proyectos o acciones que promuevan la igualdad de género en los diferentes ámbitos sociales en los que se desarrollan (SEP,2017).

Al igual que en el nivel educativo de la primaria, la temática Valoración de la Diversidad, no Discriminación e Interculturalidad sigue constituyendo un pilar para la educación en la perspectiva de género en la secundaria. Aquí se les proporcionan los conocimientos sobre las instituciones, leyes y programas que se han elaborado en México para prevenir y eliminar la discriminación. Desde la perspectiva de género el conocimiento de estas acciones institucionales les permite a los alumnos comprender la igualdad de género en términos jurídico, porque estas leyes y programas son coadyuvantes o complementarios de las leyes que protegen los derechos de las mujeres (SEP, 2017, p. 178)

Asimismo esta temática se orienta a lograr que los alumnos realicen prácticas educativas positivas que contribuyan a desarrollar una cultura incluyente e intercultural (SEP, 2017, p. 178). Desde un enfoque de género, el impulso para la construcción de una cultura incluyente es una acción a favor de la igualdad de género en términos sustantivos porque en este tipo de cultura no cabe la discriminación y el trato diferenciado para con las mujeres.

\subsection{La educación en la perspectiva de género en el nivel educativo de primaria}

Tabla 1: Educación y género. Nivel educativo primaria.

\begin{tabular}{|c|c|c|c|}
\hline $\begin{array}{c}\text { Grado } \\
\text { Escolar }\end{array}$ & Tema /1 & Aprendizajes Esperados /1 & $\begin{array}{c}\text { Objetivos de aprendizaje para el } \\
\text { ODS 5: Igualdad de género /2 }\end{array}$ \\
\hline $4^{\circ}$ & $\begin{array}{c}\text { Igualdad y perspectiva de } \\
\text { género }\end{array}$ & $\begin{array}{c}\text { Comprende que mujeres y hombres } \\
\text { tienen iguales derechos y que la } \\
\text { discriminación afecta a la } \\
\text { dignidad de las personas }\end{array}$ & $\begin{array}{c}\text { El/ la alumno/ a es capaz de } \\
\text { observar e identificar la } \\
\text { discriminación de género. }\end{array}$ \\
\hline $5^{\circ}$ & $\begin{array}{c}\text { Igualdad y perspectiva de } \\
\text { género }\end{array}$ & $\begin{array}{c}\text { Reconoce situaciones de } \\
\text { desigualdad de género y realiza } \\
\text { acciones a favor de la equidad }\end{array}$ & $\begin{array}{c}\text { El/la alumno/ a comprende los } \\
\text { niveles de desigualdad de } \\
\text { género dentro de su } \\
\text { propio país y cultura. }\end{array}$ \\
\hline $6^{\circ}$ & Igualdad y perspectiva de \\
género & $\begin{array}{c}\text { Distingue características } \\
\text { naturales y sociales entre } \\
\text { hombres y mujeres y sus }\end{array}$ & $\begin{array}{c}\text { El/ la alumno/a comprende los } \\
\text { conceptos de género, igualdad de } \\
\text { género y discriminación de género } \\
\text { y conoce sobre todas las formas } \\
\text { de discriminación, violencia y } \\
\text { desigualdad de género. }\end{array}$ \\
\hline
\end{tabular}

Fuentes: /1 Aprendizajes Clave para la Educación Integral. Plan y programas de estudio para la educación básica SEP, 2017, México. $/ 2$ Educación para los Objetivos de Desarrollo Sostenible. Objetivos de Aprendizaje, UNESCO, 2017, París. Francia.

Los aprendizajes esperados de la enseñanza de la temática Igualdad y perspectiva de género, muestran que en $4^{\circ}$ grado de primaria la educación en la perspectiva de género se centra en los siguientes aspectos: a) teniendo como marco la dignidad como un atributo inherente a todo ser humano se aborda la igualdad de derechos de mujeres y hombres, así como la discriminación como una práctica que no toma en cuenta este valor intrínseco que tiene todo ser humano (SEP, 2017,p. 450). Este aprendizaje esperado se complementa con el objetivo de aprendizaje conductual número cuatro de los Objetivos de aprendizaje para alcanzar el ODS 5 que se centra en la identificación de la discriminación de género (UNESCO, 2017, 20)

El aprendizaje esperado en el $5^{\circ}$ grado de primaria se enfoca al reconocimiento de la desigualdad de género (SEP, 2017, p. 450). Este aprendizaje también se complemente con el objetivo de aprendizaje cognitivo número tres, de los Objetivos de aprendizaje para alcanzar el ODS 5 porque contempla el conocimiento de la desigualdad de género desde una perspectiva amplia. Es decir 
plantea la importancia de que el alumno conozca los niveles de desigualdad de género dentro de su país y cultura en comparación con las normas mundiales tomando en cuenta la cultura que tiene cada país (UNESCO, 2017, p. 20).

En el $6^{\circ}$ grado de primaria el aprendizaje esperado coincide con el objetivo de aprendizaje cognitivo número uno de los Objetivos de aprendizaje para alcanzar el ODS 5 porque ambos proporcionan elementos para comprender el género como una construcción sociocultural (SEP, 2017, p. 450) (UNESCO, 2017, p. 20)

\subsubsection{La educación en la perspectiva de género en el nivel educativo de secundaria}

Tabla 2. Educación y género. Nivel educativo secundaria

\begin{tabular}{|c|c|c|c|}
\hline $\begin{array}{l}\text { Grado } \\
\text { Escolar }\end{array}$ & Tema /1 & Aprendizajes Esperados /1 & $\begin{array}{c}\text { Objetivos de aprendizaje para el ODS } 5: \\
\text { Igualdad de género } / 2\end{array}$ \\
\hline $1^{\circ}$ & $\begin{array}{l}\text { Igualdad y } \\
\text { perspectiva de } \\
\text { género }\end{array}$ & $\begin{array}{c}\text { Analiza situaciones de la vida social y } \\
\text { política de México a la luz del } \\
\text { derecho a la igualdad }\end{array}$ & $\begin{array}{c}\text { El/la a alumno/a conoce las oportunidades } \\
\text { y beneficios que ofrece la igualdad y la } \\
\text { participación plena del género en la } \\
\text { legislación y la gobernanza incluida la } \\
\text { asignación de presupuesto público, el } \\
\text { mercado laboral y la toma de decisiones } \\
\text { públicas y privadas. }\end{array}$ \\
\hline $2^{\circ}$ & $\begin{array}{l}\text { Igualdad y } \\
\text { perspectiva de } \\
\text { género }\end{array}$ & $\begin{array}{c}\text { Analiza las implicaciones de la equi- } \\
\text { dad de género en situaciones cerca- } \\
\text { nas a la adolescencia: } \\
\text { amistad, noviazgo, estudio }\end{array}$ & $\begin{array}{c}\text { El/ la alumno/ a es capaz de reconocer y } \\
\text { cuestionar la percepción tradicional de los } \\
\text { roles de género desde una perspectiva } \\
\text { crítica a la vez que respeta el } \\
\text { anclaje cultural. }\end{array}$ \\
\hline $3^{\circ}$ & $\begin{array}{l}\text { Igualdad y } \\
\text { perspectiva de } \\
\text { género }\end{array}$ & $\begin{array}{c}\text { Promueve la igualdad de género en } \\
\text { sus relaciones cotidianas y lo integra } \\
\text { como criterio para } \\
\text { valorar otros espacios }\end{array}$ & $\begin{array}{l}\text { El/la alumno/a es capaz de conectarse } \\
\text { con otros para trabajar juntos en aras } \\
\text { del fin de la violencia y discriminación de } \\
\text { género, empoderar a aquellos que todavía } \\
\text { carezcan de poder y fomentar el respeto y } \\
\text { la plena igualdad en todos los niveles. }\end{array}$ \\
\hline
\end{tabular}

Fuentes: 11 Aprendizajes Clave para la Educación Integral. Formación Cívica y Ética. Educación secundaria. Plan y programas de estudio, orientaciones didácticas y sugerencias de evaluación, SEP, 2017, México. /2 Educación para los Objetivos de Desarrollo Sostenible. Objetivos de Aprendizaje, UNESCO, 2017, París, Francia

En el $1^{\circ}$ grado de secundaria el aprendizaje esperado se sintetiza en el conocimiento y análisis del derecho a la igualdad (SEP, 2017, p. 178). Este aprendizaje se complementa con el objetivo de aprendizaje cognitivo número cuatro de los Objetivos de aprendizaje para alcanzar el ODS 5 que contempla el conocimiento de las oportunidades y beneficios que brinda la igualdad y la participación plena de los géneros en diferentes ámbitos de la sociedad (UNESCO, 2017,p. 20).

En el $2^{\circ}$ grado de secundaria el aprendizaje esperado se centra en conocer y analizar las implicaciones que tiene la equidad de género en las relaciones que establecen en la adolescencia como son: «amistad, noviazgo, estudio». (SEP, 2017, p. 179). Este aprendizaje se complementa con el objetivo de aprendizaje socioemocional número uno de los Objetivos de aprendizaje para alcanzar el ODS 5 que plantea la importancia de desarrollar en el alumno el pensamiento y la perspectiva crítica para que les permita cuestionar la percepción tradicional de los roles de género (UNESCO, 2017, p .20).

En el $3^{\circ}$ grado de secundaria, el aprendizaje esperado implica que el alumno asuma y ejerza la igualdad de género en los diferentes ámbitos donde se desarrolla a la vez que impulsa y promueve este tipo de igualdad (SEP, 2017,p. 180). Este aprendizaje también se complementa con el objetivo de aprendizaje socioemocional número tres de los Objetivos de aprendizaje para alcanzar el ODS 5 porque plantea desarrollar en el alumno la capacidad de conectarse y trabajar con otros para erradicar 
la violencia y la discriminación del género, lo cual constituye una forma de impulsar y concretizar la igualdad de género (UNESCO, 2017, p. 20).

\section{DISCUSIÓN Y CONCLUSIONES}

Los resultados del análisis de la temática de Igualdad y perspectiva de género del Plan de Estudios para la Educación Básica de la SEP (2017) muestran que los aprendizajes esperados del estudio de esta temática se complementan con los objetivos de aprendizaje planteados en los Objetivos de aprendizaje para alcanzar el ODS 5 de la UNESCO (2017).

Teniendo como referente a estos últimos objetivos resulta pertinente que la educación en la perspectiva de género de esta propuesta curricular incluya y desarrolle otros aprendizajes socioemocionales y conductuales que están presentes en los Objetivos de aprendizaje para alcanzar el ODS 5 de la UNESCO (2017), para que los alumnos puedan adoptar los valores y las actitudes que requiere el ejercicio de la igualdad y equidad de género.

\section{REFERENCIAS BIBLIOGRÁFICAS}

Instituto Nacional para las Mujeres (2017), Ley general para la igualdad entre mujeres y hombres, México: INMUJERES. Recuperado de www.cedoc.inmujeres.gob.mx/documentos_download/lgimh.pdf.

ONU Mujeres (2015), Igualdad de género, México: Entidad de las Naciones Unidas para la igualdad de género y el empoderamiento de las mujeres. Recuperado de: www.igualdaddegenero.unam.mx/wp-content/uploads/.../onu-mujeres-igualdad-equidad.pd.

Secretaria de Educación Pública, (2017a). Aprendizajes clave para la educación Integral. Planes y Programas de estudio, México: SEP. Recuperado de: www.aprendizajesclave.sep.gob.mx/.../APRENDIZAJES_CLAVE_PARA_LA_EDUCAC.

Secretaria de Educación Pública, (2017b). Aprendizajes clave para la educación integra. Formación cívica y ética. Educación Secundaria. Plan y programas de estudio, orientaciones didácticas y sugerencias de evaluación, México: SEP. Recuperado de: www.aprendizajesclave.sep.gob.mx/.../fcye/1-LpM-sec-Formacion-Civica-y-etica.pdf.

Secretaria de Educación Pública, (2017 c). Modelo educativo para la educación obligatoria. Educar para la libertad y creatividad, México: SEP. Recuperado de: https://www.gob.mx/cms/.../Modelo_Educativo_para_la_Educacio_n_Obligatoria.pdf.

UNESCO (2017 a). Educación para los objetivos de Desarrollo Sostenible. Objetivos de aprendizaje, Paris: UNESCO. Recuperado de: www.unesdoc.unesco.org/images/0025/002524/252423s.pdf.

UNESCO (2014 b). Indicadores UNESCO de cultura para el desarrollo. Manual Metodológico, Paris: UNESCO. Recuperado de: www.es.unesco.org/creativity/sites/creativity/files/iucd_manual_metodologico_1.pdf.

UNESCO (2017c). Plan de acción de la UNESCO, para la prioridad «lgualdad de Género» 2014-2021, Paris: UNESCO, Recuperado de: www.unesdoc.unesco.org/images/0022/002272/227222s.pdf. 